\title{
A predicted miR-27a-mediated network identifies a signature of glioma
}

\author{
SHUXU YANG ${ }^{1 *}$, KUN WANG $^{2 *}$, CONG QIAN $^{1}$, ZHENGFEI SONG $^{2}$, PEIYU PU ${ }^{3,4}$, ANLING ZHANG $^{3,4}$, \\ WEI WANG ${ }^{5}$, HUANJIANG NIU ${ }^{1}$, XINWEI LI $^{1}$, XUCHEN QI $^{1}$, YINXIN ZHU ${ }^{1}$ and YIRONG WANG ${ }^{1}$ \\ ${ }^{1}$ Department of Neurosurgery, Sir Run Run Shaw Hospital, Medical College, Zhejiang University; \\ ${ }^{2}$ Department of Neurosurgery, Hangzhou Xiasha Hospital, Sir Run Run Shaw Hospital, Medical College, \\ Zhejiang University, Hangzhou 310016; ${ }^{3}$ Department of Neurosurgery, Tianjin Medical University General Hospital; \\ ${ }^{4}$ Laboratory of Neuro-oncology, Tianjin Neurological Institute, Tianjin 300052; ${ }^{5}$ Department of Pharmacy, \\ Sir Run Run Shaw Hospital, Medical College, Zhejiang University, Hangzhou 310016, P.R. China
}

Received April 10, 2012; Accepted June 18, 2012

DOI: 10.3892/or.2012.1955

\begin{abstract}
The dysregulation of physiological microRNA (miRNA) activity has been shown to play an important role in gliomagenesis. In a previous study, using microRNA arrays and glioma tissues found that miR-27a was upregulated, which was also identified in the glioma cell lines and samples by quantitative real-time polymerase chain reaction (qRT-PCR). In this study, in order to explore the potential roles of miR-27a in the progression of glioma, we first utilized text-mining of PubMed abstracts with natural language processing (NLP) to identify 1,168 glioma-related molecules. In addition, miR-27a targets predicted by computational methods were integrated with the results from NLP analysis, followed by Gene Ontology (GO), pathway and network analysis. We identified 33 hub genes by overlap calculation and demonstrated that miR-27a may be involved in the progression of glioma through adherens junction, focal adhesion, the neurotrophin signaling pathway, the MAPK signaling pathway, the transforming growth factor- $\beta$ (TGF- $\beta$ ) signaling pathway, cytokine-cytokine receptor interactions, the p53 signaling pathway, the apoptotic signaling pathway, as well as others. Our data may provide researchers with a better understanding of the mechanisms of the miR-27a-target network in glioma initiation and progression.
\end{abstract}

\section{Introduction}

Gliomas are the most frequently observed brain tumors, with glioblastoma multiforme (GBM) being the most common and

Correspondence to: Dr Yirong Wang, Department of Neurosurgery, Sir Run Run Shaw Hospital, Medical College, Zhejiang University, 3 Qingchun Road East, Hangzhou 310016, P.R. China

E-mail: wang.yr@163.com

*Contributed equally

Key words: glioma, miR-27a, signature aggressive form in adults (1). Despite the major therapeutic improvements achieved with the combination of neurosurgery, chemotherapy and radiotherapy, the prognosis and survival rate for patients with GBM remains extremely low (2). Therefore, there is an urgent need for innovative and reliable diagnostic or prognostic biomarkers and new therapeutic strategies. Gene signatures that can characterize the heterogeneity of glioma would provide a molecular basis for the pathological or clinical characteristics and the regulatory pathways and networks that are unique to glioma subtypes and would lead to the identification of molecular fingerprints for a more guided treatment plan.

microRNAs (miRNAs) comprise a large group of endogenous non-coding RNAs that can either block mRNA translation or negatively regulate miRNA stability, and therefore play a crucial role in the regulation of gene expression (3). A number of studies have analyzed miRNA expression profiles in glioblstoma and have identified several deregulated miRNAs (4-6). Furthermore, alterations in miR-levels have been implicated in the deregulation of critical players in major cellular pathways, modifying the differentiation, proliferation and survival of tumor cells. For instance, miR-7 and miR-221/222 have been shown to be involved in the activation of the Akt and epidermal growth factor receptor (EGFR) signaling pathways, while miR-34a is a key downstream regulator of p53 (7-10). As cancer-related molecular signatures are definitely not one or two, but rather a series of factors, the comprehensive and systematic analysis of miRNA-target genes is of great importance in glioma treatment and may provide a unique method for the diagnosis and prognosis of glioma, in addition to providing novel targets for the development of therapeutic strategies for glioma.

In the present study, we found that miR-27a is upregulated in human glioma. Furthermore, we systematically analyzed the miR-27a-predicted target genes related to the glioma by a series of data-filtering, including Gene Ontology (GO), pathway and network analyses. Our results provide novel insights into our understanding of the role and mechanism of miR-27a in gliomagenesis and provide a potential therapeutic strategy for glioma treatment in the future. 


\section{Materials and methods}

Cell culture and human glioma samples. The human U251, U87, LN229, LN308 and A172 glioblastoma cell lines were purchased from the Institute of Biochemistry and Cell Biology, Chinese Academy of Sciences. All cells were maintained at $37^{\circ} \mathrm{C}$ in a $5 \% \mathrm{CO} 2$ incubator in Dulbecco's modified Eagle's medium (DMEM) (Gibco, Carlsbad, CA, USA) supplemented with 10\% fetal bovine serum (Invitrogen, Carlsbad, CA, USA).

The human glioma samples were obtained from the Department of Neurosurgery, Sir Run Run Shaw Hospital, Medical College, Zhejiang University, Hangzhou, China. Informed consent was obtained from all patients diagnosed with glioblastoma prior to obtaining the specimens. The samples were freshly resected during surgery and immediately frozen in liquid nitrogen for subsequent total RNA extraction.

RNA extraction and quantitative real-time polymerase chain reaction ( $q R T-P C R$ ). The total RNA was isolated from cultured cells, human glioma specimens, or normal brain tissues (NBTs) using TRIzol ${ }^{\circledR}$ reagent (Invitrogen) according to the manufacturer's instructions. qRT-PCR was performed in triplicate in the ABI 7500HT fast real-time PCR System (Applied Biosystems) and normalized to U6 and glyceraldehyde 3-phosphate dehydrogenase (GAPDH) endogenous control. Total RNA from NBTs was used as the control. The miR-27a levels were measured with the TaqMan microRNA assay kit in accordance with the manufacturer's instructions (Applied Biosystems).

Natural language processing (NLP) of glioma studies. We conducted a search in PubMed (Medline), in an attempt to cover all studies published with the following keywords: glioma (title). All the sets of genes and proteins associated with the keywords were identified and added to a list, followed by gene mention tagging, using a biomedical named entity recognizer (ABNER; an open source tool for automatically tagging genes, proteins and other entity names in text, http:// pages.cs.wisc.edu/ bsettles/abner/) (11) and conjunction resolution. In the present study, the gene symbol in the Entrez gene database of NCBI prevailed as previously described $(12,13)$. A flow chart of the NLP analysis is shown in Fig. 1.

Prediction of miRNA target genes. The analysis of miR-27a predicted targets was determined using three independent softwares (14): PicTar2005(http://pictar.mdc-berlin.de/cgi-bin/ PicTar_vertebrate.cgi); miRandaV5(http://www.ebi.ac.uk/ enright-srv/microcosm/htdocs/targets/v5/) and TargetScan 5.1(http://www.targetscan.org/) .

GO analysis. GO analysis was conducted with the GSEABase R package (http://www.r-project.org/) statistical Platform (15). miR-27a targets were then classified into three major groups: biological process, cellular component and molecular function.

Pathway analysis. miR-27a targets were mapped to the Kyoto Encyclopedia of Genes and Genomes (KEGG) pathway database by using GenMAPP v2.1, and the p-value was calculated for each enriched pathway (16).

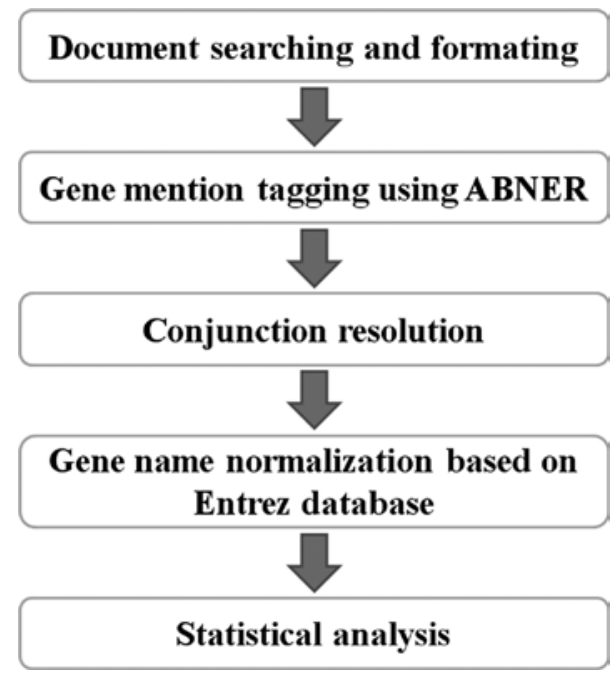

Figure 1. Flow chart of the natural language processing (NLP) analysis of glioma.

Table I. Gene network analysis including three types of interaction: enzyme-enzyme interaction, protein-protein interaction and gene expression interaction.

ECrel enzyme-enzyme interaction, indicating two enzymes catalyzing successive reaction steps

PPrel protein-protein interaction, such as binding and modification

GErel gene expression interaction, indicating correlation between transcription factor and target gene product

Network analysis. We also integrated three different interactions: i) protein interaction, gene regulation and protein modification in the KEGG database; ii) the existing highthroughput experiments, such as the protein-protein interaction confirmed by yeast two-hybrid; iii) the already mentioned interaction between genes. In brief, we downloaded the pathway data from the KEGG database and analyzed the genomic interaction between the genes by using the KEGGSOA (http:// www.bioconductor.org/packages/2.4/bioc/html/KEGGSOAP. html) R package (http://www.r-project.org/), including three types of interaction: enzyme-enzyme relation, protein-protein interaction and gene-expression interaction (17) (Table I).

The protein-protein interaction data were downloaded from the MIPS database (http://mips.helmholtz-muenchen.de/ proj/ppi/) (18). For the afore-mentioned interaction we used algorithm co-citation in the PubMed abstracts: we analyzed a gene term and all its co-occuring term variants within the sentences in the PubMed abstracts and calculated the frequency of the co-citation gene. Subsequently, we performed a statistical amalysis as described in the NLP analysis. Finally, the network was displayed by using the Medusa software.

Integrative analysis of miR-27a target genes and NLP. The overlap between the miR-27a target genes predicted by bioinformatic tools and the glioma related-genes obtained from 
the NLP analysis was calculated. A gene network analysis was subsequently performed.

Statistical analysis. In the analysis of miR-27a expression, all tests were carried out using SPSS Graduate Pack 11.0 statistical software (SPSS, Chicago, IL). Descriptive statistics including the mean \pm SE along with one-way ANOVAs were used to determine the significant differences. $\mathrm{P}<0.05$ was considered to indicate a statistically significant difference.

In the NLP analysis, the frequency of each gene occurrence was calculated. The higher the frequency of genes the greater the likelihood of an association between glioma and a certain gene. The total number of references in the PubMed database was recorded as ' $N$ '. The frequency of genes and glioma in the PubMed database was denoted by ' $m$ ' and ' $n$ ', respectively. The simultaneous occurrence of a gene and the disease was denoted as ' $\mathrm{k}$ '. Then by using hypergeometric distribution, we calculated the probability of frequency greater than ' $\mathrm{k}$ ' co-citation at the completely random conditions. $\mathrm{P}<0.01$ was considered to indicate a statistically significant difference. We used the following formula:

$$
\begin{aligned}
& p=1-\sum_{i=0}^{k-1} p(i \mid n, m, N) \\
& p(i \mid n, m, N)=\frac{n !(N-n) ! m !(N-m) !}{n-i ! i ! n-m ! N-n-m+i ! N !}
\end{aligned}
$$

\section{Results}

miR-27a is overexpressed in human glioma. We have previously demonstrated that miR-27a is upregulated in glioblastoma by miRNA arrays (data not shown). To further identify the expression of miR-27a in glioma, we performed TaqMan-based real-time stem-loop RT-PCR analyses using fresh human glioma samples and cell lines. We selected five glioblastoma cell lines, including U251, U87, LN229, LN308 and A172 for testing and used NBT as the normal control. Our data showed that miR-27a was significantly upregulated in all of these glioblastoma cell lines, compared to NBT (Fig. 2A). A similar result was observed in the glioma tissues (Fig. 2B), and all of these samples showed a significant increase (5 to 14 fold) in miR-27a expression. Taken together, our results demonstrated that miR-27a was abnormally overexpressed both in human glioma samples and cell lines.

Integrative analysis of miR-27a target genes and NLP results. After proving the upregulation of miR-27a in glioma, we futher investigated the potential role of miR-27a and its targets in glioma development. We used three computational algorithms: TargetScan4.0, PicTar and miRanda, which are commonly used to predict miRNA targets, and identified 1,884 miR-27a target genes. In addition, the abstracts and titles of 11,150 primary studies were identified for initial reviewing using the search strategies already mentioned and a total of 1,168 glioma-related genes were subsequently identified.

To further explore the potential mechanism of miR-27a, we categorized the integrative results of NLP analysis and miR-27a targets in $\mathrm{GO}$ according to biological process, cellular
Table II. All of the genes obtained from integration of NLP analysis and potential miR-27a targets were categorized in GO, according to biological process, cellular component and

\begin{tabular}{|c|c|c|}
\hline Term & Count & p-value \\
\hline \multicolumn{3}{|l|}{ Biological process } \\
\hline Developmental processes & 61 & 0 \\
\hline Death & 28 & $2.08 \mathrm{E}-10$ \\
\hline Cell cycle and proliferation & 28 & $1.36 \mathrm{E}-06$ \\
\hline Stress response & 24 & $2.58 \mathrm{E}-05$ \\
\hline Cell adhesion & 14 & 0.0007227 \\
\hline Cell organization and biogenesis & 29 & 0.0010944 \\
\hline Protein metabolism & 37 & 0.0016411 \\
\hline Cell-cell signaling & 9 & 0.0021401 \\
\hline Signal transduction & 43 & 0.0126941 \\
\hline RNA metabolism & 30 & 0.0629269 \\
\hline Other biological processes & 65 & 0.0873043 \\
\hline Other metabolic processes & 27 & 0.2618713 \\
\hline Transport & 23 & 0.2850757 \\
\hline DNA metabolism & 3 & 0.6768301 \\
\hline \multicolumn{3}{|l|}{ Cellular component } \\
\hline Nucleus & 48 & 0.0043901 \\
\hline Plasma membrane & 33 & 0.0072076 \\
\hline Cytoskeleton & 14 & 0.0222964 \\
\hline Cytosol & 7 & 0.0245927 \\
\hline Extracellular matrix & 6 & 0.0285326 \\
\hline Other cellular component & 64 & 0.0704202 \\
\hline Non-structural extracellular & 18 & 0.0817679 \\
\hline Other cytoplasmic organelle & 5 & 0.3191817 \\
\hline Mitochondrion & 9 & 0.3869073 \\
\hline Other membranes & 59 & 0.6113681 \\
\hline ER/Golgi & 9 & 0.7024601 \\
\hline Translational apparatus & 1 & 0.8080292 \\
\hline \multicolumn{3}{|l|}{ Molecular function } \\
\hline Kinase activity & 21 & $2.85 \mathrm{E}-06$ \\
\hline Other molecular function & 118 & 0.0003563 \\
\hline Transcription regulatory activity & 18 & 0.0028955 \\
\hline Cytoskeletal activity & 9 & 0.0202878 \\
\hline Signal transduction activity & 35 & 0.1061263 \\
\hline Enzyme regulator activity & 7 & 0.2699902 \\
\hline Nucleic acid binding activity & 23 & 0.3250427 \\
\hline Transporter activity & 7 & 0.7310064 \\
\hline
\end{tabular}
molecular function.

component and molecular function (Table II). Subsequent to the pathway analysis, there were 50 pathways available, out of which eight signaling pathways were significant $(\mathrm{p}<0.01)$ (Table III): the adherens junction pathway (NLK, INSR, SNAI1, SMAD2, MET, CDC42, FYN, EGFR, FGFR1, YES1; $\mathrm{p}=2.25 \mathrm{E}-06$ ); the focal adhesion pathway (GRB2, PDPK1, XIAP, MET, PDGFRA, PXN, IGF1, CDC42, FYN, RAP1B, EGFR, FN1; p=0.0006989); the neurotrophin signaling pathway (GRB2, IRS1, MAPK14, BAX, CDC42, FASLG, RAP1B, MAPK7, NGFRAP1); the MAPK signaling pathway 


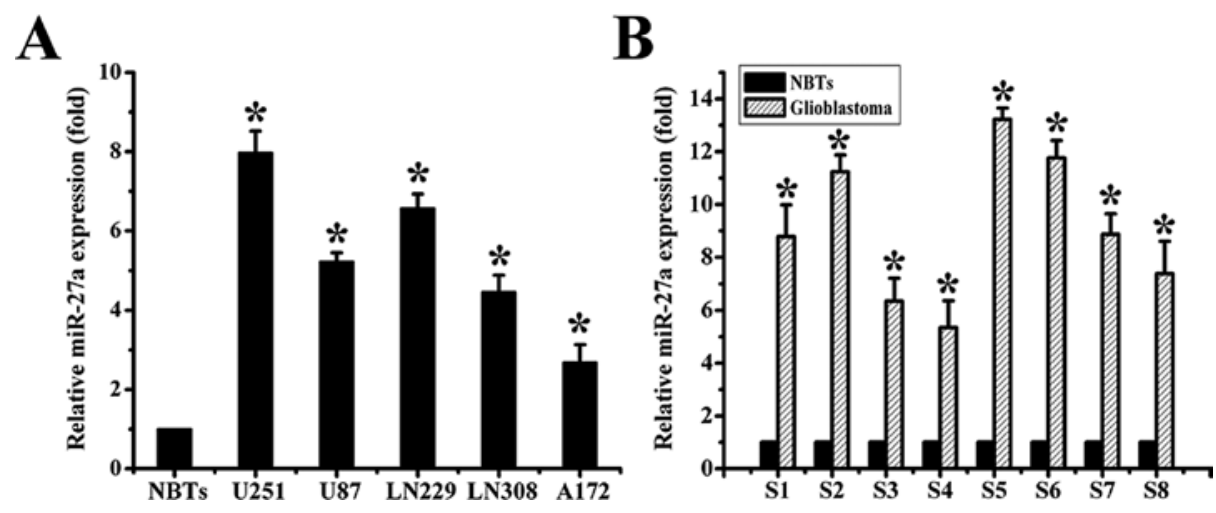

Figure 2. miR-27a is overexpressed in glioma cell lines and samples. (A) miR-27a expression in glioma cells (U251, U87, LN229, LN308 and A172) and normal brain tissue (NBT) by qRT-PCR. (B) qRT-PCR analysis showed that glioblastoma tissues expressed much higher levels of miR-27a compared with the NBT. Data represen the means \pm SE of thee replicates; ${ }^{*} p<0.05$.

Table III. Pathway analysis of the genes obtained from integration of the NLP analysis and potential miR-27a targets ${ }^{\mathrm{a}}$.

\begin{tabular}{|c|c|c|c|}
\hline Title & Count & p-value & Genes \\
\hline Adherens junction & 10 & $2.25 \mathrm{E}-06$ & $\begin{array}{l}\text { NLK, INSR, SNAI1, SMAD2, MET, CDC42, FYN, EGFR, } \\
\text { FGFR1, YES1 }\end{array}$ \\
\hline Focal adhesion & 12 & 0.0006989 & $\begin{array}{l}\text { GRB2, PDPK1, XIAP, MET, PDGFRA, PXN, IGF1, } \\
\text { CDC42, FYN, RAP1B, EGFR, FN1 }\end{array}$ \\
\hline Neurotrophin signaling pathway & 9 & 0.0010528 & $\begin{array}{l}\text { GRB2, IRS1, MAPK14, BAX, CDC42, FASLG, RAP1B, } \\
\text { MAPK7, NGFRAP1 }\end{array}$ \\
\hline MAPK signaling pathway & 14 & 0.0010648 & $\begin{array}{l}\text { NLK, GRB2, CDC25B, PDGFRA, MAPK14, NF1, CDC42, } \\
\text { FASLG, RAP1B, MAPK7, EGFR, MAP2K4, FGFR1, FGF1 }\end{array}$ \\
\hline TGF- $\beta$ signaling pathway & 7 & 0.0017426 & SMAD2, ID4, SMAD5, SP1, BMPR2, ID3, RPS6KB1 \\
\hline Cytokine-cytokine receptor interaction & 13 & 0.0024092 & $\begin{array}{l}\text { CXCL2, MET, PF4, IL6R, PDGFRA, IL10, FASLG, CSF3, } \\
\text { BMPR2, EGFR, CSF1, IL24, KITLG }\end{array}$ \\
\hline p53 signaling pathway & 6 & 0.0024798 & PMAIP1, BAX, IGF1, CASP8, CDK6, BBC3 \\
\hline Apoptosis & 6 & 0.008848 & XIAP, CFLAR, BAX, FADD, FASLG, CASP8 \\
\hline
\end{tabular}

${ }^{a}$ There were 50 pathways available. Among them, eight signaling pathways were significant $(\mathrm{p}<0.01)$.

(NLK, GRB2, CDC25B, PDGFRA, MAPK14, NF1, CDC42, FASLG, RAP1B, MAPK7, EGFR, MAP2K4, FGFR1, FGF1; $\mathrm{p}=0.0010648)$; the transforming growth factor- $\beta(\mathrm{TGF}-\beta)$ signaling pathway (SMAD2, ID4, SMAD5, SP1, BMPR2, ID3, RPS6KB1; $p=0.0017426)$; the cytokine-cytokine receptor interaction (CXCL2, MET, PF4, IL6R, PDGFRA, IL10, FASLG, CSF3, BMPR2, EGFR, CSF1, IL24, KITLG; p=0.0024092); the p53 signaling pathway (PMAIP1, BAX, IGF1, CASP8, CDK6, BBC3; $\mathrm{p}=0.0024798)$; and the apoptotic pathway (XIAP, CFLAR, BAX, FADD, FASLG, CASP8; p=0.008848).

The gene network can not only directly reflect the relationship between genes, but also the stability of gene regulatory networks. The highly connected hub genes often play an important role in the stability of the network. Since the hub genes are the core of gene regulation and can affect the majority of genes, a hub gene is generally believed to be of greater importance than another normal gene. In the network analysis of the integrative targets, the connectivity of growth factor receptor-bound protein 2 (GRB2) was also the highest among all 33 hub genes obtained in the miR-27a target network analysis (a total of six genes; z-test, $\mathrm{p}=0.0010559$ ): CD28, EGFR, FGFR1, IRS1, MET and PDGFRA (Fig. 3).

The overlap of the miR-27a target genes and the gliomarelated genes obtained from NLP analysis was calculated and 44 overlap genes that are not only associated with the development and progression of glioma but are also the potential miR-27a target genes were obtained in this integrative analysis (Fig. 4; Table IV).

\section{Discussion}

Gliomas are the most frequently observed brain tumors, with GBM being the most common and aggressive form in adults (1). Unfortunately, the underlying molecular mechanisms resulting in gliomagenesis and local invasion remain obscure and are the major obstruction to finding novel therapeutic strategies. 

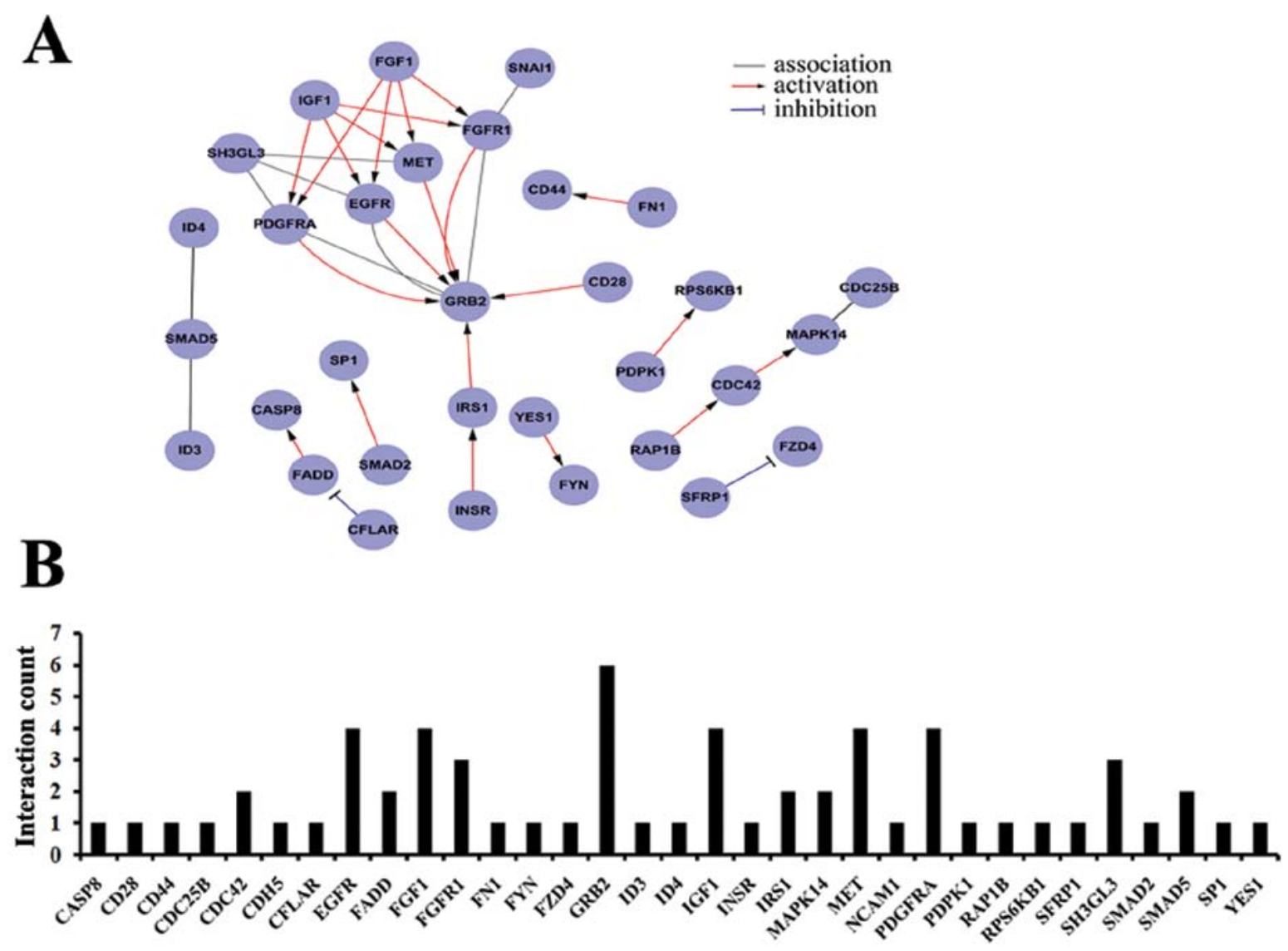

Figure 3. (A) Network analysis of glioma-related genes indentified in the natural language processing (NLP) analysis. The network can reflect the relationship between genes from a situation as a whole. Grey, association; red, activation; blue, inhibition. (B) Connectivity analysis of the glioma-related genes. The connectivity of GRB2 was the highest among the total glioma-related genes ( $\mathrm{z}$-test, $\mathrm{p}<0.01)$.

Erb signaling pathway

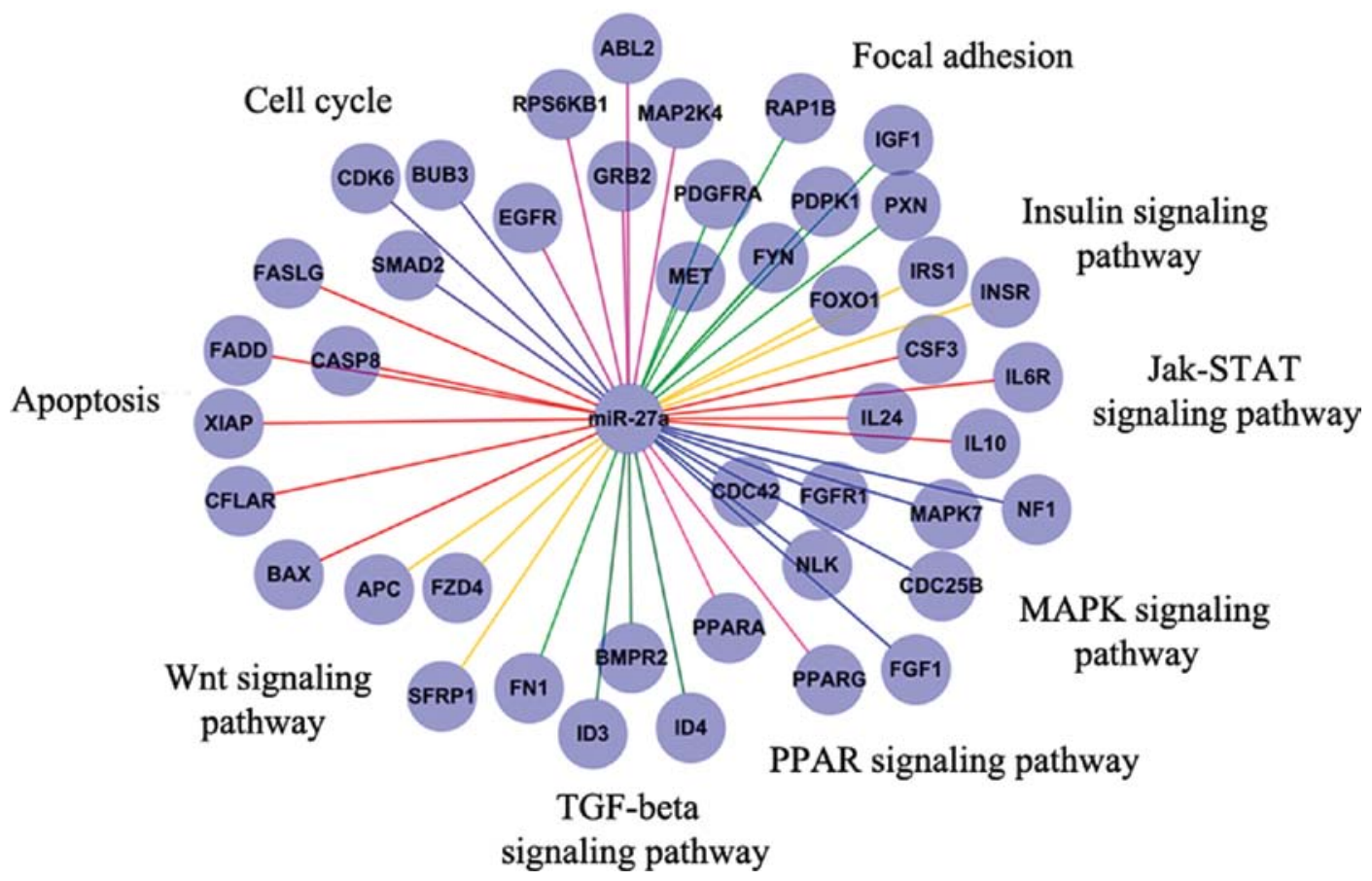

Figure 4. Integrative-analysis of miR-27a target genes and the natural language processing (NLP) results. A total of 44 overlap genes and their functional pathways that are not only associated with the molecular mechanism of glioma but also the potential miR-27a target genes were obtained in this final integrative analysis. 
Table IV. Integrative analysis of miR-27a target genes and the NLP results ${ }^{\mathrm{a}}$.

\begin{tabular}{|c|c|c|c|}
\hline Targets & PubMed count & p-value & Gene description \\
\hline FASLG & 20 & 0 & Fas ligand (TNF superfamily, member 6) \\
\hline FADD & 8 & 0 & Fas (TNFRSF6)-associated via death domain \\
\hline CASP8 & 10 & 0 & Caspase 8 , apoptosis-related cysteine peptidase \\
\hline BAX & 13 & 0 & BCL2-associated X protein \\
\hline NF1 & 18 & 0 & Neurofibromin 1 \\
\hline IL10 & 18 & 0 & Interleukin 10 \\
\hline IL24 & 7 & 0 & Interleukin 24 \\
\hline PDPK1 & 8 & 0 & 3-Phosphoinositide-dependent protein kinase 1 \\
\hline EGFR & 227 & 0 & $\begin{array}{l}\text { Epidermal growth factor receptor (erythroblastic leukemia } \\
\text { viral (v-erb-b) oncogene homolog, avian) }\end{array}$ \\
\hline CDK6 & 6 & 0 & Cyclin-dependent kinase 6 \\
\hline PPARG & 16 & $1.94 \mathrm{E}-08$ & Peroxisome proliferator-activated receptor gamma \\
\hline FGFR1 & 6 & $2.23 \mathrm{E}-06$ & Fibroblast growth factor receptor 1 \\
\hline PXN & 5 & $9.25 \mathrm{E}-06$ & Paxillin \\
\hline XIAP & 5 & $2.15 \mathrm{E}-05$ & X-linked inhibitor of apoptosis \\
\hline PDGFRA & 4 & 0.0002609 & Platelet-derived growth factor receptor, alpha polypeptide \\
\hline CDC25B & 3 & 0.0002675 & Cell division cycle 25 homolog B (S. pombe) \\
\hline PPARA & 4 & 0.0015537 & Peroxisome proliferator-activated receptor alpha \\
\hline SLTM & 1 & 0.0062219 & SAFB-like, transcription modulator \\
\hline RPS6KB1 & 2 & 0.024244 & Ribosomal protein S6 kinase,70kDa, polypeptide 1 \\
\hline CFLAR & 2 & 0.038005 & CASP8 and FADD-like apoptosis regulator \\
\hline NLK & 1 & 0.040756 & Nemo-like kinase \\
\hline FZD4 & 1 & 0.044739 & Frizzled homolog 4 (Drosophila) \\
\hline ID4 & 1 & 0.044739 & $\begin{array}{l}\text { Inhibitor of DNA binding } 4 \text {, dominant negative } \\
\text { helix-loop-helix protein }\end{array}$ \\
\hline BUB3 & 1 & 0.054624 & Budding uninhibited by benzimidazoles 3 homolog (yeast) \\
\hline RAP1B & 1 & 0.060506 & RAP1B, member of RAS oncogene family \\
\hline ID3 & 1 & 0.07985 & $\begin{array}{l}\text { Inhibitor of DNA binding } 3 \text {, dominant negative } \\
\text { helix-loop-helix protein }\end{array}$ \\
\hline ABL2 & 1 & 0.085576 & $\begin{array}{l}\text { V-abl Abelson murine leukemia viral oncogene homolog } 2 \\
\text { (arg, Abelson-related gene) }\end{array}$ \\
\hline SFRP1 & 1 & 0.11919 & Secreted frizzled-related protein 1 \\
\hline MAPK7 & 1 & 0.12284 & Mitogen-activated protein kinase 7 \\
\hline MAP2K4 & 1 & 0.15859 & Mitogen-activated protein kinase kinase 4 \\
\hline CSF3 & 1 & 0.16557 & Colony stimulating factor 3 (granulocyte) \\
\hline BMPR2 & 1 & 0.18275 & $\begin{array}{l}\text { Bone morphogenetic protein receptor, type II } \\
\text { (serine/threonine kinase) }\end{array}$ \\
\hline FGF1 & 1 & 0.20455 & Fibroblast growth factor 1 (acidic) \\
\hline IL6R & 1 & 0.21115 & Interleukin 6 receptor \\
\hline FOXO1 & 1 & 0.22093 & Forkhead box $\mathrm{O} 1$ \\
\hline SMAD2 & 1 & 0.32089 & SMAD family member 2 \\
\hline CDC42 & 1 & 0.40925 & Cell division cycle 42 (GTP binding protein, 25kDa) \\
\hline IRS1 & 1 & 0.42502 & Insulin receptor substrate 1 \\
\hline FN1 & 1 & 0.43215 & Fibronectin 1 \\
\hline FYN & 1 & 0.48397 & FYN oncogene related to SRC, FGR, YES \\
\hline INSR & 1 & 0.49774 & Insulin receptor \\
\hline APC & 1 & 0.51621 & Adenomatous polyposis coli \\
\hline GRB2 & 1 & 0.58868 & Growth factor receptor-bound protein 2 \\
\hline IGF1 & 1 & 0.73593 & Insulin-like growth factor 1 (somatomedin C) \\
\hline
\end{tabular}

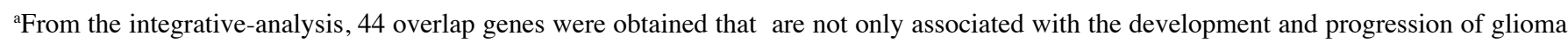
but are also potential miR-27a target genes. 
Our approach links putative targets to a function and succeeds not only in identifying multiple targets but also in uncovering an entire network under miRNA control.

miR-27a has been studied by several researcheres. Recently, Zhao et al proved that the downregulation of miR-27a inhibits the proliferation of gastric cancer cells in vitro and in vivo, increases the sensitivity of gastric cancer cells to drugs, and can increase the accumulation or decrease the amount of adriamycin in gastric cancer cells (19). Wang et al reported that the upregulation of miR-27a contributes to the malignant transformation of human bronchial epithelial cells induced by the SV40 small T antigen (20). Ma et al showed that the inhibition of miR-27a suppressed the growth, colony formation and migration of pancreatic cancer cells (21). Zhang et al demonstrated that the downregulation of miR-27a increases the sensitivity of esophageal cancer cells to P-glycoprotein-related and P-glycoprotein-non-related drugs, and can promote ADR-induced apoptosis, accompanied by the increased accumulation and decreased in the amount of ADR (22). However, the precise molecular mechanisms of miR-27a involved in glioma are unclear.

In the present study, we focused on the miR-27a expression and the predicted signature of a miR-27a-mediated network for glioma. We first identified the abnormal overexpression of a miR-27a in glioma by qRT-PCR. To explore the potential mechanisms involved, we carried out NLP analysis, and identified 1,168 genes related to the development and progression of glioma. Given that the biological significance of miRNA deregulation relies on the effect upon their target-proteincoding genes, we analyzed the predicted targets of miR-27a. To date, computational methods have been widely used for the prediction of miRNA target genes. It has been shown that the union of miRNA target genes predicted by three computational algorithms (miRanda, PicTar, and TargetScan) is one of the strategies that demonstrate the highest sensitivity (14). In our study, this strategy predicted a total of 1,884 unique gene symbols targeted by miR-27a. Then the NLP analysis and miR-27a putative targets were integrated and categorized in $\mathrm{GO}$, followed by pathway and network analyses, including the tumor-related genes, CDK6, SMAD2, FASLG, FADD, BAX, CASP8, FOXO1, PPARG and MET. Some of these targets have already been verified by miRNA functional experiments, such as PPARG (23), FOXO1 (24) and FADD (25). A total of 50 pathways were obtained in the miR-27a target pathway analysis and some of them have already been reported to be involved in the development and progression of glioma. Pathways such as the adherens junction (26), the focal adhesion (27), the neurotrophin signaling pathway (28), the MAPK signaling pathway (29), the TGF- $\beta$ signaling pathway (30), the cytokine-cytokine receptor interaction (31), the p53 signaling pathway (32) and the apoptotic signaling pathway (33) play an important role in the molecular mechanisms of glioma $(\mathrm{p}<0.01)$. Other pathways, such as the ErbB signaling pathway (34), the insulin signaling pathway (35), the PPAR signaling pathway (36), the Wnt signaling pathway (37), the cell cycle (38) and the Jak-STAT signaling pathway (39) are also involved in glioma progression. Since the highly connected hub genes are the core of gene regulation and affect the majority of genes, it is generally believed that hub genes are of greater importance than other normal genes and often play a crucial role in the stability of the network. We also carried out connectivity analysis in this study, and GRB2 had the highest connectivity (a total of six genes; $\mathrm{z}$-test, $\mathrm{p}=0.0010559$ ), of all the 33 hub genes obtained in the miR-27a target network analysis (Fig. 3). Other hub genes, such as EGFR (40), PDGFRA (32), FADD (41) and MET (42) are also associated with cancer. Cancer-related biomarkers are definitely a group or a series of factors. The joint detection of these 33 hub genes may potentially enable doctors to identify and select high-risk patients for effective adjuvant therapy in order to improve the results of patients with glioma.

A total of 33 hub genes were identified in the final integrative analysis of NLP and miR-27a targets. Our results suggest that miR-27a may plays an important role in the development and progression of glioma through the adherens junction, focal adhesion, neurotrophin signaling, MAPK signaling, TGF- $\beta$ signaling, cytokine-cytokine receptor interaction, p53 signaling and the apoptotic signaling pathway, as well as others. Our integrative study also suggests that targeting those hub genes and their pathways may be an effective strategy to control cell proliferation in glioma.

In conclusion, the present study provides new insights into the role of miR-27a in human brain tumors. We propose that a systematic analysis of the miR-27a-targets in glioma may be an effective tool for the early diagnosis, prognosis and prediction of the therapeutic response of the individual. Our results may help researchers predict the molecular mechanisms of miR-27a in the development and progression of glioma. The identification of these molecular pathways involved in the malignant biological behavior of glioma may play a prominent role in establishing rational therapeutic approaches.

\section{Acknowledgements}

The authors wish to express their gratitude to Shanghai Sensichip Co., Ltd. for the bioinformatic analysis.

\section{References}

1. Louis DN, Ohgaki H, Wiestler OD, Cavenee WK, Burger PC, Jouvet A, Scheithauer BW and Kleihues P: The 2007 WHO classification of tumours of the central nervous system. Acta Neuropathol 114: 97-109, 2007.

2. Behin A, Hoang-Xuan K, Carpentier AF and Delattre JY: Primary brain tumours in adults. Lancet 361: 323-331, 2003.

3. Ambros V: The functions of animal microRNAs. Nature 431: 350-355, 2004.

4. Ciafre SA, Galardi S, Mangiola A, Ferracin M, Liu CG, Sabatino G, Negrini M, Maira G, Croce CM and Farace MG: Extensive modulation of a set of microRNAs in primary glioblastoma. Biochem Biophys Res Commun 334: 1351-1358, 2005.

5. Chan JA, Krichevsky AM and Kosik KS: MicroRNA-21 is an antiapoptotic factor in human glioblastoma cells. Cancer Res 65: 6029-6033, 2005.

6. Dong H, Siu H, Luo Li, Fang X, Jin L and Xiong M: Investigation gene and microRNA expressionin in glioblastoma. BMC Genomics 3: S16, 2010.

7. Kefas B, Godlewski J, Comeau L, Li Y, Abounader R, Hawkinson M, Lee J, Fine H, Chiocca EA, Lawler S and Purow B: microRNA-7 inhibits the epidermal growth factor receptor and the Akt pathway and is downregulated in glioblastoma. Cancer Res 68: 3566-3572, 2008.

8. Zhang J, Han L, Ge Y, Zhou X, Zhang A, Zhang C, Zhong Y, You Y, Pu P and Kang C: miR-221/222 promote malignant progression of glioma through activation of the Akt pathway. Int J Oncol 36: 913-920, 2010.

9. Li Y, Guessous F, Zhang Y, et al: microRNA-34a inhibits glioblastoma growth by targeting multiple oncogenes. Cancer Res 69: 7569-7576, 2009. 
10. Chang TC, Wentzel EA, Kent OA, et al: Transactivation of miR-34a by $\mathrm{p} 53$ broadly influences gene expression and promotes apoptosis. Mol Cell 26: 745-752, 2007.

11. Settles B: ABNER: an open source tool for automatically tagging genes, proteins and other entity names in text. Bioinformatics 21: 3191-3192, 2005.

12. Smith L, Tanabe LK, Ando RJ, et al: Overview of BioCreative II gene mention recognition. Genome Biol 9: S2, 2008.

13. Morgan AA, Lu Z, Wang X, et al: Overview of BioCreative II gene normalization. Genome Biol 9: S3, 2008.

14. Lewis BP, Shih IH, Jones-Rhoades MW, Bartel DP and Burge CB: Prediction of mammalian microRNA targets. Cell 115: 787-798, 2003.

15. Morgan M, Falcon S and Gentleman R: GSEABase: Gene set enrichment data structures and methods. $\mathrm{R}$ package version 1.4.0

16. Dahlquist KD, Salomonis N, Vranizan K, Lawlor SC and Conklin BR: GenMAPP, a new tool for viewing and analyzing microarray data on biological pathways. Nat Genet 31: 19-20, 2002.

17. Ogata H, Goto S, Fujibuchi W and Kanehisa M: Computation with the KEGG pathway database. Biosystems 47: 119-128, 1998.

18. Mewes HW, Albermann K, Heumann K, Liebl S and Pfeiffer F: MIPS: a database for protein sequences, homology data and yeast genome information. Nucleic Acids Res 25: 28-30, 1997.

19. Zhao X, Yang L and Hu J: Down-regulation of miR-27a might inhibit proliferation and drug resistance of gastric cancer cells. J Exp Clin Cancer Res 30: 55, 2011.

20. Wang Q, Li DC, Li ZF, et al: Upregulation of miR-27a contributes to the malignant transformation of human bronchial epithelial cells induced by SV40 small T antigen. Oncogene 30: 3875-3886, 2011.

21. Ma Y, Yu S, Zhao W, Lu Z and Chen J: miR-27a regulates the growth, colony formation and migration of pancreatic cancer cells by targeting Sprouty2. Cancer Lett 298: 150-158, 2010.

22. Zhang H, Li M, Han Y, Hong L, Gong T, Sun L and Zheng X: Down-regulation of miR-27a might reverse multidrug resistance of esophageal squamous cell carcinoma. Dig Dis Sci 55 $2545-2551,2010$

23. Kim SY, Kim AY, Lee HW, Son YH, Lee GY, Lee JW, Lee YS and Kim JB: miR-27a is a negative regulator of adipocyte differentiation via suppressing PPARgamma expression. Biochem Biophys Res Commun 392: 323-328, 2010.

24. Guttilla IK and White BA: Coordinate regulation of FOXO1 by miR-27a, miR-96 and miR-182 in breast cancer cells. J Biol Chem 284: 23204-23216, 2009.

25. Chhabra R, Adlakha YK, Hariharan M, Scaria V and Saini N: Upregulation of miR-23a-27a-24-2 cluster induces caspasedependent and -independent apoptosis in human embryonic kidney cells. PLoS One 4: e5848, 2009.

26. Yiin JJ, Hu B, Jarzynka MJ, Feng H, Liu KW, Wu JY, Ma HI and Cheng SY: Slit2 inhibits glioma cell invasion in the brain by suppression of Cdc42 activity. Neuro Oncol 11: 779-789, 2009.

27. Brockschmidt A, Trost D, Peterziel H, et al: KIAA1797/FOCAD encodes a novel focal adhesion protein with tumour suppressor function in gliomas. Brain 135: 1027-1041, 2012.

28. Chou TT, Trojanowski JQ and Lee VM: Neurotrophin signal transduction in medulloblastoma. J Neurosci Res 49: 522-527, 1997.
29. Festa M, Capasso A, D'Acunto CW, Masullo M, Rossi AG, Pizza C and Piacente S: Xanthohumol induces apoptosis in human malignant glioblastoma cells by increasing reactive oxygen species and activating MAPK pathways. J Nat Prod 74: 2505-2513, 2011.

30. Eichhorn PJ, Rodón L, Gonzàlez-Juncà $\mathrm{A}$, et al: USP15 stabilizes TGF- $\beta$ receptor I and promotes oncogenesis through the activation of TGF- $\beta$ signaling in glioblastoma. Nat Med 18: 429-435, 2012.

31. Liu KW, Hu B and Cheng SY: Platelet-derived growth factor receptor alpha in glioma: a bad seed. Chin J Cancer 30: 590-602, 2011.

32. Stegh AH and DePinho RA: Beyond effector caspase inhibition: Bcl2L12 neutralizes p53 signaling in glioblastoma. Cell Cycle 10: 33-38, 2011.

33. Akasaki Y, Liu G, Matundan $\mathrm{HH}, \mathrm{Ng} \mathrm{H}$, Yuan X, Zeng Z, Black KL and Yu JS: A peroxisome proliferator-activated receptor-gamma agonist, troglitazone, facilitates caspase- 8 and -9 activities by increasing the enzymatic activity of protein-tyrosine phosphatase-1B on human glioma cells. J Biol Chem 281: 6165-6174, 2006.

34. Gondi CS, Dinh DH, Klopfenstein JD, Gujrati M and Rao JS: MMP-2 downregulation mediates differential regulation of cell death via ErbB-2 in glioma xenografts. Int J Oncol 35: 257-263, 2009.

35. Eisinger DA and Ammer H: Epidermal growth factor treatment switches $\delta$-opioid receptor-stimulated extracellular signalregulated kinases 1 and 2 signaling from an epidermal growth factor to an insulin-like growth factor-1 receptor-dependent mechanism. Mol Pharmacol 79: 326-335, 2011.

36. Isaac AO, Kawikova I, Bothwell AL, Daniels CK and Lai JC: Manganese treatment modulates the expression of peroxisome proliferator-activated receptors in astrocytoma and neuroblastoma cells. Neurochem Res 31: 1305-1316, 2006.

37. Liu C, Tu Y, Sun X, Jiang J, Jin X, Bo X, Li Z, Bian A, Wang X, Liu D, Wang Z and Ding L: Wnt/beta-Catenin pathway in human glioma: expression pattern and clinical/prognostic correlations. Clin Exp Med 11: 105-112, 2011.

38. Besson A and Yong VW: Mitogenic signaling and the relationship to cell cycle regulation in astrocytomas. J Neurooncol 51: 245-264, 2001.

39. Tu Y, Zhong Y, Fu J, Cao Y, Fu G, Tian X and Wang B: Activation of JAK/STAT signal pathway predicts poor prognosis of patients with gliomas. Med Oncol 28: 15-23, 2011.

40. Zhang Y, Xing X, Zhan H, Yu Q and Chen J: EGFR inhibitor enhances cisplatin sensitivity of human glioma cells. J Huazhong Univ Sci Technolog Med Sci 31: 773-778, 2011.

41. Ho IA, Ng WH and Lam PY: FasL and FADD delivery by a glioma-specific and cell cycle-dependent HSV-1 amplicon virus-enhanced apoptosis in primary human brain tumors. Mol Cancer 9: 270, 2010.

42. Xie Q, Bradley R, Kang L, et al: Hepatocyte growth factor (HGF) autocrine activation predicts sensitivity to MET inhibition in glioblastoma. Proc Natl Acad Sci USA 109: 570-575, 2012. 